\title{
Improving The Supervision Process: Trust, Presence, Empowerment And Recognition
}

\section{Stephen D Benigno}

Trust, presence, empowerment and the recognition of success are four administrative strategies that can facilitate a successful implementation of the new instructional supervision models. The recent initiatives involving the effective and formative supervision of instruction in the public school systems essentially require more than just an effective model of supervision. The relationship between the administration and the instructional staff that has traditionally involved simply an observation, a walk-through, check sheet and has resulted in a summative and terminal evaluation of instruction, will definitely require a paradigm shift. The new models emphasize a collaborative and communicative relationship between the teachers and the school administration. The utilization of the four administrative strategies may facilitate the collaboration and communication necessary to achieve the successful Implementation and delivery of the new models of instructional supervision. The development and improvement of instruction and student learning outcomes in our schools will be dependent on that collaborative and communicative relationship.

New initiatives related to the formative and developmental supervision of instruction have recently surfaced within school districts across the country. Research has indicated that the teacher is the most influential factor in the academic achievement of the student. "The past decade has confirmed what we intuitively know-teachers are the most important in-school factor that influences student achievement" (Nye, Konstantopoulos, Hedges 2004). "We need to streamline supervision and evaluation so that principals can spend their time doing what will make the most difference: quickly and efficiently keeping tabs on what is really happening in classrooms, giving teachers constant feedback, making fair judgments about teacher performance and getting teams invested in improving student learning and focused on results" (Minnici,2014). With this new emphasis on teacher evaluations, states and school districts are embracing formative instructional supervision designs that can enhance the development of the classroom teacher through improved observation and data collection strategies. One of the strategic initiatives is the clinical supervision model of instruction. This process involves the developmental supervision of instruction through a sequence of observations and collaborations between the teacher and the supervising administrator. While the organization and design of the model are important, the success of this initiative is critically dependent on a functionally sound relationship between the supervisor and the teacher. "Changes in evaluation processes and outcomes require not just new tools and procedures but significant changes in norms and beliefs" (Minnici 2014). A collegial relationship based on confidence, trust and collaboration must be established prior to implementing the supervision strategy. "Much of the instructional supervision that takes place in the public school system is summative and specifically related to terminal teacher evaluations" (Benigno, 2016). Glickman, Gordon and Ross-Gordon (2009), have indicated that "historically, supervision has involved inspection of teachers and is usually viewed as an instrument for controlling teachers" (p.7). "An opportunity for collaboration between the supervisor and the teacher with respect to the evaluation process is usually not adequately addressed in these expedited models of teacher 
evaluation" (Benigno, 2016). Acknowledging the importance of that collegial and collaborative relationship between the administration and the instructional staff is paramount in the successful implementation of the clinical supervision model. It is extremely important that school administrators understand their role in creating an environment in the schools that is conducive to developing professional and collegial relationships. It is critical that administrative teams develop specific strategies that can address these needs.

Building trust between the administration and the instructional staff isn't an easy endeavor. Culture, climate and the existing norms can influence the outcome of any effort to build trust, "norms can make or break new initiatives, new employees, or new teachers" (Gruenert, Whitaker 2015) but addressing the trust factor will facilitate the development and enhancement of collaboration between the supervisors and the teacher. The trust relationship between the administration and the instructional staff can be enhanced through collegial conversation, informal dialogue and shared experiences. Conversations during faculty, department, Special Education and student study team meetings that involve a give and take between faculty members and the administration will stimulate dialogue and help to eliminate that barrier between the teachers and the administration. "The capacity to engage in honest and disclosing talk cannot be underestimated in professional learning communities" (Lieberman, Miller 2008). Casual conversations between the administration and faculty members may also stimulate a connection, which down the road, will facilitate the instructional supervision exercise. Administrative interest and attention to the teachers and their endeavors will demonstrate an "ethic of care," (Noddings 2005). This ethic of care may facilitate the development of a collegial and collaborative relationship with the administration and the teachers that can go a long way in developing trust. "In an effective culture, members are confident that they can share their professional struggles with anyone else in the culture without invalidating their work" (Gruenert, Whitaker 2015) Administrative transparency with respect to communication is also critical. Teachers must be able to understand the expectations of the administration. It is essential that the administration conveys truthful and coherent messages, provides active disclosure and that the commitments to the instructional staff are maintained with fidelity. This transparency is an important characteristic that should be demonstrated during the orientation, observations and conferences of the supervision process.

There is a conceptual difference between simply being present and presence. Administrative personnel are often present during the school day but fail to exude presence. Presence is the active participation in the moment, the engagement with people or an activity. Standing in the hall way watching the students pass is not presence, doing a walk through without providing feedback to the teachers is not presence. Presence should be demonstrated by the administration through active supervision, engaging visibility and accessible availability. The supervisor who exudes presence participates in activities, collaborates with the students and the teachers, attends activities that are sometimes "off the radar" and can often be seen eating lunch with the students and the teachers. These strategic behaviors can be paramount in developing a relationship between the administration and instructional staff that will enhance the supervision process. When teachers are asked to complete an assignment, participate in a professional learning opportunity or represent to a stakeholder, it is important that authentic and constructive feedback concerning the opportunity be provided in a timely fashion. That feedback, if completed with fidelity and consistency will solidify the perception that you have a genuine interest in the success of the instructional staff and that you are demonstrating a presence with respect to the assignment or expectation. Active supervision entails authentic 
and strategic instructional supervision strategies that involve collaborative and engaging interactions with students, parents, teachers and classified personnel in the building. An active supervisor meets the teachers at the door in the morning and bids them goodbye at night, the available administrator attends the professional learning opportunities and participates in the activities. The administrator that walks through classrooms, patrols the halls, has a cup of coffee in the faculty room and stops to talk to teachers, parents and students is authentically available.

Empowerment is a tool that if used strategically, can enhance the instructional supervision process. The administrator who develops a conceptual knowledge of the instructional expertise, leadership and experience in the building can use that knowledge to empower those teachers to facilitate the instructional supervision process. Teachers who are new or novice to the profession often receive more direct supervision from the administration while your veteran, proficient teachers, are allowed to develop their instructional prowess without directional interference. This tailored instructional supervision strategy will empower many of the "expert" teachers to become facilitators, mentors and leaders. This empowerment strategy will be embraced and recognized by the teachers as an opportunity to participate in the shared leadership of the building. This shared leadership can translate into a successful implementation of the clinical supervision of instruction model. The expert teachers can lead the initiative and facilitate the successful roll out of the instructional supervision plan.

The recognition of teacher success elevates morale in the school, acknowledges authentic accomplishment and serves notice that the administration knows what's going on in the building. This recognition will create confidence and encourage the instructional staff to be more receptive of new initiatives presented by the administration. The new teacher evaluation model will be more readily embraced if morale is high and teachers feel self-actualized. An "authentic acknowledgement" of individual and group success will help develop an intrinsic desire in the teachers to develop and progress in the profession. Teacher accomplishment should be rewarded formatively and frequently throughout the course of the instructional year. Teachers should be afforded an opportunity to participate in instructional and noninstructional endeavors throughout the school year. These opportunities should be rewarded by the administration through formal and informal processes. Formal acknowledgement or reward could be in the form of a monetary or ceremonial recognition through a formal presentation process or could be of an informal nature that simply involves the teacher and an administrator in an intimate setting. Either form is acceptable if the recognition is authentic and sincere in the delivery of the message. Multiple opportunities should be afforded to teachers during the course of the year. Each of the opportunities should result in some kind of recognition for the teachers. If this concept of teacher recognition is followed with fidelity by the administration, teacher morale, performance and commitment should improve incrementally.

The new supervision models are not the panacea or the utopian connection between the teacher, the administration and improved instruction. The personalization of the process is the key to the successful implementation of a process which requires collaboration, communication, facilitation and cooperation. Effective schools are successful in mobilizing both the academic core of schools-teaching and learning-as well as practices which encourage affective relationships between adults (Ingersoll, 2003). The new models of instructional supervision require the administration to develop skills above and beyond the transactional strategies that were utilized with previous models of instructional supervision. Strategies to 
develop teacher ownership of the process through the building of trust and the empowerment of the teacher will transform the instructional supervision process into an effective tool that can formatively improve instructional and teacher performance.

\section{References}

Benigno, Stephen. (2016) A viable solution to implementing effective instructional supervision. Journal of Education and Learning; Vol. 5, No. 1 Canadian Center of Science and Education

Glickman, Carl, D., Gordon, Stephen P., Ross-Gordon, Jovita M. (2014) Supervision and Instructional Leadership, A Developmental Approach. Pearson Education Inc.

Gruenert, Steve, Whitaker, Todd. (2015) School culture rewired, how to define, assess, and transform it. Copyright @ ASCD (2015)

Ingersoll, R. M. (2003) Who controls teachers work? Power and accountability in America's schools. Cambridge, MA: Harvard University Press.

Leiberman, Ann, Miller, Lynne (2008) Developing capacities, Teachers in Professional Communities, chapter 2 pg. 15. New York: Teachers College Press

Minnici, Angela. (2014) The mind shift in teacher evaluation. American Educator. Spring 2014

Noddings, Nel. The Challenge to Care in Schools, New York: Teachers College Press, 2005

Nye, Barbara, Konstantopoulos, Spyros, Hedges Larry V. (2004). How large are teacher effects. Educational Evaluation and Policy Analysis Vol. 26, No.3, pp.237-25 